\title{
Genomic variation in Austrian and Leningrad populations of snail Arianta arbustorum L.
}

\author{
O. Bondareva ${ }^{1 *}$, A. Kasianov ${ }^{2}$, N. Abramson ${ }^{1}$ \\ ${ }^{1}$ Zoological Institute RAS, St.-Petersburg, Russia \\ ${ }^{2}$ Vavilov institute of general genetics, Moscow, Russia \\ *e-mail: olga.v.bondareva@gmail.com
}

Key words: invasion, genetic paradox, Arianta arbustorum L., RNA-seq

Motivation and Aim: The invasion occurs in extremely short periods, which allows us to consider them as "natural experiments", which provide a unique opportunity to observe the ecological and evolutionary processes in real time. Among the species of terrestrial ecosystems, one example of the extremely successful invasion of Russia in the NorthWest region of recent years is the tree snail Arianta arbustorum (Linneus, 1758).

Methods and Algorithms: In order to analyze the overall variability that potentially could lead to the success of $A$. arbustorum snail migration, we made a comparative analysis of single nucleotide polymorphysm (SNP calling) in transcripts of individuals from different populations: from Austria, which are part of the original range of A. arbustorum, and from the vicinity St. Petersburg, where this species was discovered relatively recently. We assembled a transcript using all samples with the following statistical parameters: Contig N50: 713, Median contig length: 372, Average contig: 564.45. After the functional annotation of the transcriptome with Transdecoder programm, there were 54 thousand contiguous verified, which we used as a reference for the search for single nucleotide substitutions.

Results: The final analysis confirmed the much lower variability of individuals from the Leningrad Region in comparison with the Austrian population, that we showed before using COI partial sequence. The main characteristics of the substitutions remain unchanged between the populations - a much larger number of transitions than transversions, the prevalence of substitutions in the 3 positions of the codon compared with the replacement in other positions and the predominance of the number of synonymous substitutions over non-synonymous ones.

Conclusion: The data obtained during the meta-analysis confirm our hypothesis that the settlement of the Leningrad region occurred by a group of founders with low haplotypic variability, who then proceeded to expansive reproduction.

Acknowledgements: This study was conducted in Zoological Institute RAS under the research theme No. AAAA-A17-117042410167-2 and supported by the RFBR 16-3400958 and RFBR 15-29-02526. 